\title{
Etude De La Pertinence Des IG Comme Outil De Différenciation Des Produits De Terroir: CAS DE l'IGP Argane Dans La Ville d'Agadir Et Regions
}

\author{
El Bakkouri Bouchra, (PhD Student) \\ Souaf Malika, (PES) \\ Elwazani Youssef, (HDR) \\ Ibn Zohr University/ENCG Agadir, Morocco
}

doi: 10.19044/esj.2016.v12n31p207 URL:http://dx.doi.org/10.19044/esj.2016.v12n31p207

\begin{abstract}
The differentiation of local products is considered as a necessity nowadays given the standardization flows are increasing. This differentiation can be established especially through the Distinctive Signs of Origin and Quality (DSOQ). Our work has shown through the example of the PGI (Protected Geographical Indication) Argane, that GIs are a relevant tool for the differentiation of local products if they (GIs) are placed in a favorable context to their application.

Our paper answers the following question: "To what extent geographical indications are meant as an effective tool for differentiation of local products: case of the PGI Argane in the city of Agadir and regions?

To do so, we used an exploratory qualitative study through semi-structured interviews with a sample of people representing different stakeholders of Agadir and its regions, preceded of course by a literature review on the different key concepts.
\end{abstract}

Keywords: Geographical Indications, differenciation, terroir Products, IGP Argane.

\section{Résumé}

La différenciation des produits de terroir s'avère aujourd'hui une nécessité compte tenu du flux de standardisation qui ne cesse d'augmenter. Cette différenciation peut s'établir notamment à travers les signes distinctifs de l'origine et de la qualité (SDOQ). Notre travail a montré à travers l'exemple de l'IGP Argane que les IG constituent un outil pertinent pour la différenciation des produits de terroir si elles (les IG) sont placées dans un contexte favorable à leur application. 
Notre papier répond à la problématique suivante: « Dans quelle mesure les indications géographiques constituent-elles un moyen et un outil efficace de différentiation des produits de terroir: cas de l’IGP Argane dans la ville d’Agadir et régions?

Pour se faire, nous nous sommes basés sur une étude qualitative à vocation exploratoire à travers des entretiens semi-directifs auprès d'un échantillon de personnes qui représentent les différentes parties prenantes de la ville d’Agadir et régions, précédée bien entendu, par une revue de la littérature sur les différents concepts clés.

Mots clés: Indications Géographiques, différenciation, produits de terroir, IGP Argane

\section{Introduction}

La mesure des critères de choix des consommateurs bénéficie d’un intérêt particulier et important de la part des chercheurs et des praticiens. Elle permet aux décideurs de bien comprendre le comportement des consommateurs, et d'adapter, par conséquent, leurs offres de produits selon les marchés (Dekhili, Sirieix, 2013). Aujourd'hui, les consommateurs cherchent des produits de qualité. Cette notion de la qualité intègre dans sa définition deux critères importants (ADS40, 2015): l'absence de risques pour la santé et la recherche d'authenticité. La naissance de ces deux critères est la résultante de l'alimentation qui devient de plus en plus banalisée. La globalisation des produits élaborés en masse par les grands groupes agroalimentaires multinationaux (dont la stratégie est basée essentiellement sur la standardisation, les économies d'échelle et le formatage du comportement des consommateurs) est à la base de cette banalisation (ILBERT, TEKELIOGLU, et TOZANLI, 2009).

Pour échapper à la règle de standardisation, les produits de terroir cherchent à se différencier de leurs homologues standards présents sur le même marché. Cette différenciation peut s'établir à travers les Signes Distinctifs de l’Origine et de la Qualité (SDOQ). Ces derniers obéissent à un cahier de charges strict et précis, et très contraignant pour les producteurs. Cela leur garantit, en contre partie, la mise sur le marché d'un produit final typique, de qualité et original pour le consommateur (Chambolle \& Eric, 2003), ce qui, selon Courvoisier (2009), constitue actuellement une alternative rassurante et savoureuse à une production mondialisée et générique de masse.

Ces signes d’identification (SI) liés à l'origine ont pour objectif de protéger et de valoriser un bassin de production par la spécification d'un

${ }^{40}$ Agence de Développement Social : http://www.ads.gov.ma/ 
produit en lien avec une origine géographique à travers sa définition, ses conditions et ses moyens de production (Filippi \& Triboulet, 2006).

Ils sont issus d'une évolution historique et différente selon les contextes économiques et sociaux des pays et des époques et concernent tout ce qui permet la reconnaissance et la distinction des produits recherchés par le consommateur des autres produits similaires. Il s'agit essentiellement des signes suivants (ADS, 2015):

- $\quad$ Qualité supérieure pour les labels: Label Rouge par exemple ;

- Caractéristiques consignées dans un règlement technique ou une norme pour la certification de conformité exemple de NF Agro-alimentaire ;

- Zone et mode de production particuliers pour les produits de montagne et ceux issus de l’Agriculture biologique: le label AB ;

- $\quad$ Le label écologique de l'Union Européenne destiné à promouvoir les produits ayant un faible impact sur l'environnement tout au long de leur cycle de vie: Ecolabel (Chambre de Commerce et d'Industrie Alsace, 2013)

- $\quad$ Caractère typique lié au terroir et au savoir-faire de l'Homme, pour l'Appellation d'Origine: AOP Taliouine ;

- Caractère servant à identifier un produit comme étant originaire du terroir pour l'indication géographique ici nous avons l'exemple de notre produit à savoir IGP Argane.

Les initiatives de labellisation peuvent être issues de plusieurs types d’organismes avec des objectifs propres et pouvant être très différents les uns des autres. Ces organismes sont notamment: des ONG et des entreprises (ou leurs associations professionnelles), des organismes publics ou privés qui peuvent mettre sur le marché un label de manière autonome et aussi l'Etat (Arjaliès, Hobeika, Ponsard, \& Poret, 2013).

Au Maroc, La publication de la loi $n^{\circ} 25-06$, relative aux signes distinctifs d'origine et de qualité (SDOQ) des denrées alimentaires et des produits agricoles et halieutiques, promulguée par le dahir n¹-08-56 du 23 mai 2008, qui a créé le cadre juridique indispensable qui permet la reconnaissance et la protection des produits de terroir.(MAPM, 2012), ainsi, le Ministère du Commerce, de l'Industrie et de la Mise à Niveau de l'Economie a élaboré en collaboration avec le Ministère de l'Agriculture, de Développement Rural et des Pêches Maritimes, avec l'appui d'experts marocains et de l'ADS ( Agence de développement social, qui a fait partie de la commission qui a élaboré le décret), la norme marocaine NM 08.5.090: " Corps gras d'origine animale et végétale - Huiles d'argane - Spécifications ", qui décrit les spécificités de l'huile d'argan afin de préserver l'authenticité de ce produit spécifique à son origine et d'introduire la notion de mesure et d'évaluation pour ce produit. (ADS, 2015). 
Grace à cette loi, le mot « Argane $^{41}$ » est désormais protégé, en effet, ce mot (avec E à la fin), désigne dans cet article, l’IGP Argane, qui a pour but de faire face aux usurpations et à la concurrence déloyale que connait ce secteur. Alors que lorsque nous évoquons l’huile en général, nous parlons du terme « argan ». Pour bénéficier de l’IGP Argane tout opérateur demandeur de la certification doit (AMIGHA, 2009):

- Avoir l’habilitation de NORMACERT. .

- $\quad$ Etre référencer auprès d'AMIGHA et du ministère de l'agriculture. .

- $\quad$ S’engager à respecter le cahier des charges et le plan de contrôle.

Selon (ADS, 2015), les usurpateurs exportent les graines à l'étranger et en fabriquent de l'huile pure ou frelatée à laquelle, ils collent la mention " fabriquée au Sud du Maroc ", ainsi, selon la réglementation en vigueur (IGP Argane), personne n'a en principe le droit aujourd'hui de commercialiser ou d'utiliser une marque renfermant le mot « Argane » (Romagny, 2009).

Dans ce sens, (AMIGHA ${ }^{42}$, 2009), stipule, que « Tout produit contenant en tout ou en partie Argane dans sa composition et/ou mentionnée sur son étiquetage doit utiliser obligatoirement Argane IGP », ainsi même les grandes marques de cosmétiques vendus en Europe ${ }^{43}$ comme par exemple (Pierre fabre ou L’Oréal) doivent utiliser l'huile d'argan certifiée IGP et mentionner ainsi Argane IGP dans la listes des composantes de leurs produits, car la qualité des produits de terroir joue un rôle important dans l'attractivité, ce que Amajid, EL BAKKOURI, Souaf, et Elwazani, (2015) appellent l'attractivité des produits exportés, qui influence indirectement l'image du territoire de provenance.

Notre travail porte sur l'étude de la pertinence des IG comme outil de différenciation des produits de terroir, et nous avons choisi de mettre le point sur l'IGP Argane comme cas de travail, et la ville d'Agadir et régions comme terrain d'étude. Avant d'entamer la problématique, et les questions de notre recherche, il nous parait opportun de préciser quelles sont ces régions et justifier ce choix.

Dans le dictionnaire Larousse, le mot région est définit comme «Territoire dont l'étendue variable est déterminée soit par une unité administrative ou économique, soit par la similitude du relief, du climat et de la végétation, soit par une communauté culturelle », tandis que Juillard

\footnotetext{
${ }^{41}$ Nom traditionnel du produit dans son aire géographique, Étymologie du terme Argane : Le nom trouve ses sources dans la racine berbère " erg ", il apparaît dans les écrits dès le 12ème siècle.(AMIGHA, 2009).

${ }^{42}$ Association Marocaine de l'Indication Géographique d'Huile d'Argane

${ }^{43}$ La majorité des produits de grande consommation à base d'argan ne contiennent pas plus de $2 \%$ d'huile. Les bienfaits sont dans ce cas douteux mais les bénéfices substantiels (Romagny, 2009).
} 
(1962) stipule qu’il n’existe pas une unanimité sur la définition du mot " région », et avance dans ce sens que la région est définit tantôt comme territoire marqué par une certaine uniformité naturelle, ethnique ou économique, tantôt district hérité de l'histoire et que n'épouse aucune réalité actuelle, la région est le plus souvent conçue comme une sorte de « donné », dont on s'efforce au seuil de l'étude de justifier les limites», pour lui, la région ne représente plus le domaine confidentiel de la recherche géographique ni le cadre folklorique où cohabitent des idéologies réactionnaires mais surtout des milieux d'actions économiques et sociales. Ainsi, dans cette vision de développement, et sur le plan pratique, nous voulons dire par régions, les villes et villages sur lesquels se trouvent les coopératives productrices de l'huiles d'argan et qui couvre l'ensemble des communes de l'Arganeraie de la réserve de la biosphère de l'UNESCO (RBA) 1998. Ainsi pour le cas de l'IGP Argane et notre étude, nous voulons dire par régions, les villes et/ou villages qui remplissent les conditions suivantes:

- $\quad$ Exister sur la RBA marocaine (selon l’UNESCO 1998)

- Contenir une ou plusieurs coopératives productrices de l'huile d'argan

- S’inscrire dans une logique de développement du territoire et de la régionalisation

Le tableau suivant présente l'organisation des coopératives productrices de l'huile d'argan, sur la base duquel, nous allons par la suite nommer ce que nous appelons régions.

Tableau 1: Organisation des coopératives féminines de l'huile d'argan

\begin{tabular}{|c|c|c|c|c|c|c|c|}
\hline \multicolumn{2}{|c|}{ STATUT } & \multicolumn{5}{|c|}{$\begin{array}{l}\text { ORGANISATION DE LA FILIERE DES } \\
\text { COOPERATIVES }\end{array}$} & MISSION \\
\hline \multicolumn{2}{|c|}{ Association } & \multicolumn{5}{|c|}{$\begin{array}{l}\text { Association Nationale des Coopératives Arganiéres } \\
\text { (ANCA) }\end{array}$} & $\begin{array}{l}\text { Promotion et } \\
\text { défense }\end{array}$ \\
\hline $\begin{array}{c}\text { Groupemen } \\
\mathrm{t}\end{array}$ & $\begin{array}{c}\text { GIE } \\
\text { Argan’ } \\
\text { taroudant }\end{array}$ & $\begin{array}{c}\text { GIE } \\
\text { Targanine }\end{array}$ & $\begin{array}{c}\text { GIE } \\
\text { Vitargan }\end{array}$ & $\begin{array}{c}\text { Union } \\
\text { Tifaout } \\
\text { n'ougadir }\end{array}$ & $\begin{array}{c}\text { GIE } \\
\text { Tizarga } \\
n\end{array}$ & $\underset{44}{\text { UCFA }}$ & $\begin{array}{l}\text { Promotion et } \\
\text { commerce }\end{array}$ \\
\hline $\begin{array}{c}\text { Lieu } \\
\text { d'implantati } \\
\text { on }\end{array}$ & Taroudant & Agadir & $\begin{array}{l}\text { Essaouir } \\
\text { a }\end{array}$ & Agadir & Tiznit & $\underset{\mathrm{r}}{\text { Agadi }}$ & $\begin{array}{l}\text { Promotion et } \\
\text { commerce }\end{array}$ \\
\hline $\begin{array}{l}\text { Coopérative } \\
\text { s adhérentes }\end{array}$ & 8 & 6 & 5 & 10 & 5 & 22 & Production \\
\hline $\begin{array}{l}\text { Femmes } \\
\text { adhérentes }\end{array}$ & 418 & 238 & 278 & 406 & 179 & 986 & Production \\
\hline
\end{tabular}

Source: AMIGHA (2009)

\footnotetext{
${ }^{44}$ Union des Coopératives Féminines d’Argane
} 
Durant, la phase de l'exploration, nous avons interrogé plusieurs parties prenantes, parmi lesquelles on trouve, les coopératives, les sociétés privées, les organismes publics et les consommateurs particuliers et professionnels. Tous les entretiens se sont déroulés sur la ville d'Agadir, exception faite, pour le cas de quelques coopératives et de l'organisme ANCA (qui se trouve à Ouled Berrhil), en effet, ces dernières se localisent sur des villages autres que Agadir, ainsi, nous voulons dire -sur le plan pratique- par régions :

\section{- $\quad$ Ouled Berrhil}

- $\quad$ Essaouira

- $\quad$ Tiznit

- $\quad$ Taroudant

- $\quad$ Amskroud

- $\quad$ Drarga

Nous nous intéressons dans le présent article à l'étude des indications géographiques, qui font partie des SDOQ, comme outil de différenciation des produits de terroir et plus particulièrement au cas de l'Indication Géographique Protégée Argane (IGP Argane). Nous tentons, ainsi, de répondre à la problématique suivante: Dans quelle mesure les indications géographiques constituent-elles un moyen et un outil efficace de différentiation des produits de terroir notamment l'IGP Argane dans la ville d'Agadir et régions ? Cette problématique se traduit par un ensemble de questions que nous avons formulées comme suit:

- $\quad$ Quel est l'effet des IG sur la promotion des produits de terroir?

- $\quad$ L'IGP Argane a-t-il réellement différencié l'huile d'argan dans la ville d'Agadir et régions des autres huiles d'argan non certifiées ?

- $\quad$ Quelle est la contribution de L'IGP Argane dans le positionnement de l'huile d'argan?

- $\quad$ L'IGP Argane a-t-elle contribué à la promotion de l'huile d'argan?

Pour répondre à ces questions nous avons effectué des entretiens semi directifs auprès d'un échantillon de personnes qui représentent les différentes parties prenantes dans la ville d'Agadir et régions.

Ce travail commence par une revue de la littérature sur les différents concepts clés, à savoir les produits de terroir, les IG, et la différenciation. En suite, une présentation de la méthodologie adoptée dans le traitement de notre problématique. En fin, le reste de l'article sera consacrée aux résultats, leur analyse et leur discussion avant de donner les conclusions. 


\section{La revue de la littérature}

\section{Les produits de terroir}

La question des produits de terroir est d'une grande importance. En effet, elle constitue une opportunité très prometteuse pour les producteurs afin de développer leur marché de niche notamment au travers d'un processus de labellisation (Courvoisier, 2009). En fait, ils (les produits de terroir) ont attiré l'attention d'une grande partie des consommateurs justifiant ainsi leur choix pour ces produits par des motivations (privées ou égoïstes) liées à la recherche de qualité supérieure, de l'originalité et du moindre risque pour la santé et parfois même par des motivations altruistes liées à la préservation de l'environnement et à des préoccupations envers la société (Dekhili \& Achabou, 2013).

Les produits régionaux font référence à leur terroir, terme qui veut dire « un espace géographique délimité, défini à partir d'une communauté humaine qui construit au cours de son histoire un ensemble de traits culturels distinctifs, de savoirs, et de pratiques fondé sur un système d'interactions entre le milieu naturel et les facteurs humains. Les savoir-faire mis en jeu révèlent une originalité, confèrent une typicité et permettent une reconnaissance pour les produits ou services originaires de cet espace et donc pour les hommes qui y vivent. Les terroirs sont des espaces vivants et innovants qui ne peuvent être assimilés à la seule tradition45» (Ait Kadi et Brulhet, 2010). Le terroir est, en réalité, la résultante d’interactions complexes entre des facteurs naturels (sol, climat, ...) et humains (savoirfaire, traditions,...) (Tekelioglu et al., 2013). Le terme terroir n’a pas vraiment de synonyme dans les pays anglo-saxons et ne fait pas sens (Barham, 2003). Il s’y confond souvent avec le mot territoire (Cartier, 2004; Prévost et al., 2014). Cependant, il existe bien une différence entre les deux puisque la notion de terroir se distingue de celle de territoire par le lien à un ou à des produits. En effet, le territoire peut exister en l'absence de produits, alors que le terroir est considéré comme le territoire d'un ou de plusieurs produits, qu'ils soient des biens ou des services (Prévost et al., 2014). L’importance des produits de terroir réside dans leur capacité à créer de la valeur économique. Cette valeur concerne de plus en plus de produits, de services et l'ensemble du territoire à travers notamment le tourisme, la restauration, et d'autres activités économiques (Tekelioglu et al., 2013). Selon Lagrange, Briand et Trognon (2000), ces produits permettent aux entreprises et aux filières concernées de:

\section{- $\quad$ Développer des stratégies de différenciation}

45 La définition élaborée par l'UNESCO dans le cadre des premières rencontres internationales "Planète Terroirs " suite à un travail conjoint de l'INAO et de l'INRA France. Cité par : Terroirs et origine : leçons d'une lecture croisée des expériences du Maroc et de la France pour une méditerranée durable, Mai 2010, P20. 
- $\quad$ Bénéficier ainsi des avantages liés à une situation de concurrence monopolistique et en conséquence

- $\quad$ Echapper à la baisse des prix en maîtrisant les quantités commercialisées.

Notre travail s'articule autour de l'huile d'argan, c'est l'une des huiles alimentaires et cosmétiques les plus chère au monde et est sujet de plusieurs brevets cosmétiques aux USA et en Europe, elle a été une source de revenus des habitants du sud ouest du Maroc pendant des siècles (Aboudrare, Lybbert, \& Magnan, 2009). Une telle source de revenu, doit s’accompagner d'un développement économique et social important dans son territoire d'origine dans ce sens Amajid et al., (2016) avancent que les produits de terroir, moteur du développement social et culturel des territoires, pourtant, ce n'est pas le cas. En fait, plusieurs chercheurs et scientifiques s’interrogent sur cette contradiction, Lybbert, Barrett, et Narjisse (2002) et Romagny (2009a), pour eux, l'exploitation des ressources de l'arganeraie semble un meilleur scénario de développement pour la population locale, or, cette exploitation ne se traduit nullment par la diminution de la pauvreté ou le développement de ces zones.

Néanmoins, il est à rappeler que notre objectif dans le présent article est de démontrer si la labellisation des produits de terroir via une Indication géographique permet une différenciation par rapport aux produits standards. Aprés avoir définit les produits de terroir, il convient alors de passer à la définition des indications géographiques, leur contexte ainsi que leurs objectifs.

\section{Les indications géographiques}

Pour assurer leur mise sur le marché international, les produits locaux ou de terroir doivent s'accompagner d'un façonnement de leur image. Cette image doit être forte et attractive pour garantir une certaine différenciation surtout avec la concurrence de plus en plus acharnée et la standardisation discontinue (Simenel et al., 2009). Parmi les moyens permettant ce façonnement, on trouve les indications géographiques. En effet, l'indication géographique est un label apposé sur les produits certifiés. Elle vise, comme tous les labels, à améliorer le fonctionnement d'un marché sur un bien de confiance c'est à dire un bien dont le consommateur est incapable d'évaluer par lui-même la qualité (Nelson, 1970) cité par (Arjaliès et al., 2013). Cette apposition de l'origine géographique sur le nom des produits est une pratique commerciale visant à faire reconnaître le produit par les acteurs du marché et par les consommateurs, du moins les connaisseurs (Allaire et al., 2005).

Les IG ont une longue existence, et d’origine européenne, leur principe a été développé en France. Elles (les IG) remontent au début du 
XXème siècle suite à une crise viticole importante qui imposé une réglementation de l'usage des noms géographiques.(Fournier, Verdeaux, Avril, \& Durand, 2008)

L’Indication Géographique est l'un des titres de propriété les plus anciens, cité dans l'article 1.2) de la Convention de Paris pour la protection de la propriété industrielle (1883) dénommée “Convention de Paris”(OMPI46, 2013)- en tant qu'outils de qualification et de différenciation des produits agroalimentaires et artisanaux (Allaire et al., 2005). Selon l'Accord ADPIC (Aspects des Droits de Propriété Intellectuelle qui touchent au Commerce) dans l'article 22 de la section 3 (OMC, 1994) « Les Indications Géographiques servent à identifier un produit comme étant originaire du terroir d'un pays, ou d'une région ou d'une localité de ce territoire, dans le cas où une qualité, une réputation ou une autre caractéristique déterminée du produit peut être attribuée essentiellement à cette origine géographique».

Le signalement des produits par les indications géographiques constitue un instrument qui rassure le consommateur par son effet qualitatif et reflète, ainsi, la qualité originaire du produit (Tekelioglu et al., 2013). Ces indications informent aussi sur le nom du terroir d'origine où le produit a été élaboré et permettent donc d'établir un lien entre la qualité, l'origine et la notoriété qui en découle (ILBERT et al., 2009). Selon OMPI, l’indication géographique véhicule des informations sur les caractéristiques propres à l'origine géographique du produit et fonctionne ainsi comme un différenciateur de produits sur le marché en permettant au consommateur de distinguer un produit doté des caractéristiques dues à son origine géographique de ceux qui ne le sont pas. Ainsi, l'IG joue un rôle important en facilitant la mise au point de marques pour des produits dont la qualité est basée essentiellement sur l’origine.

Les indications géographiques ont suscité un intérêt très particulier et donc des débats acharnés autour de la convention de la diversité biologique en tant qu'outil important pour la protection des savoirs locaux en lien avec la biodiversité. Ainsi, Boisvert et Caron (2007) et Downes et Laird (1999) (cité par (Boisvert \& Caron, 2010)) présentent les caractéristiques ou en d'autres termes les objectifs des indications géographiques comme étant un droit de propriété intellectuelle différent:

Les IG protègent la réputation et non l'innovation: pour le cas de l'huile d'argan par exemple c'est un produit de terroir issu d'un arbre rare et endémique dont le processus de fabrication et de production est particulier, traditionnel et collectif et qui est transmis de génération en génération. Ici,

${ }^{46}$ Organisation Mondiale de la Propriété Intellectuelle. 
l'IG a pour objectif de protéger le produit, le savoir-faire et le processus tout entier.

Elles sont illimitées dans le temps et inaliénables, c'est-à-dire inséparables des lieux d’origine des produits en question. Cela contribue à la préservation de la richesse de la population et de leurs pratiques qui sont de plus en plus à l'abandon dans le pays d'origine.

Les IG sont liées à l’origine des produits protégés et se définissent en référence au terroir de ces produits, leur mise en œuvre n'est pas contraignante (elle ne nécessite pas l'allocation de droits spéciaux que ce soit à des entités ethniques ou à des communautés locales)

Finalement, les IG servent comme une forme de protection empêchant le dévoiement du patrimoine local et l'usurpation du nom et de la notoriété de produits locaux par des acteurs extérieurs.

Après la présentation de l'indication géographique notamment sa définition, ses caractéristiques et ses objectifs, il nous semble opportun de définir la différenciation, ses aspects et ses formes.

\section{La Différenciation}

Bathelot, dans l'encyclopédie illustrée du marketing47, définit la différenciation comme « une politique produit par laquelle une entreprise va différencier son produit vis à vis de ceux de la concurrence. Elle peut se faire par le développement de caractéristiques perçues comme uniques par le consommateur ». Pour le cas de l'huile d'Argan il s'agit d'une différenciation par la qualité liée à l’origine par le biais des Indications Géographiques.

Selon Sylvander et Barjolle (2003), la notion de différenciation rejoint celle de la spécificité. En effet, le produit ne peut être différencié s’il n’a pas des caractéristiques particulières c'est à dire mesurables au sens substantiel ou intrinsèque et véritablement différentes des produits de substitution et donc perçues comme spécifique par les usagers du produit. Pour Sylvander et Lassaut (1994 cité par (Sylvander \& Barjolle, 2003)), les "produits d'origine certifiée” établissent ainsi une différence par rapport aux produits standards présents sur le même marché.

Romagny (2009), avance qu'il existe deux types de stratégies qui s’imposent aux producteurs locaux dans ce cas (l'ouverture des marchés internationaux et l'évolution des préférences des consommateurs):

- $\quad$ celles basées sur le coût (faire le même produit moins cher)

- $\quad$ et celles recherchant la différenciation. Les questions de spécificité du produit (qu'est-ce qui fait qu’il est unique?), des caractéristiques qui le

\footnotetext{
${ }^{47}$ http://www.definitions-marketing.com/definition/differenciation/. Consulté le 20/05/2016
} 
lient à son territoire et aux savoirs locaux des communautés paysannes, et surtout leur reconnaissance, deviennent alors fondamentales.

Pour nous, la première stratégie, ne constitue pas une solution innovatrice ou créative: il est facile de présenter le même produit que celui des concurrents à un prix moins cher. Par contre la deuxième stratégie qui porte sur la différenciation est une possibilité qui permet, à notre égard, de mettre en valeur le bien du pays, de la population et le bien personnel du producteur. En effet, valoriser un produit de terroir se répercute sur son pays d'origine (la valeur ajoutée revient au pays et à sa population), et sur son attractivité in-site, selon Amajid et al., (2016), la production des produits de terroirs permet le développement et la promotion de circuits touristiques thématique, créant en conséquence une offre différenciée pour les touristes intéressés par le tourisme rural et culturel. Romagny (2009) rejoint cette idée: « Avec l'IGP Argane, le Maroc affiche son intention de conserver sur son territoire une plus grande part des bénéfices issus de la vente d’huile, et d'améliorer les retombées socio-économiques positives de cette activité au niveau de la région de production des noix d'argan. » et sur le producteur local lui-même en générant des profits non négligeables.

Ainsi, pour le deuxième choix, qui est celui de la stratégie de différenciation, Hobeika, Ponssard et Poret (2013) proposent de mobiliser deux types de différenciation en l'occurrence la différenciation horizontale et la différenciation verticale. La premiére concerne les entreprises qui proposent des produits de qualité identique avec des caractéristiques différentes (dans le cas de la labellisation, il s’agit de la valorisation de ces caractéristiques différentes), tandis que la deuxième concerne, quant à elle, des biens qui ont les mêmes structures de caractéristiques avec une qualité finale différente (ou avec des quantités de caractéristiques différentes). Ici, la labellisation peut, dans certains cas, se présenter comme l'assurance d'un niveau minimal de qualité.

Au-delà de leurs définitions, Hobeika et al., (2013) proposent un tableau qui explique le lien entre la différenciation avec ses deux formes, et les labels :

Tableau 2 : Formes de différenciation et labels

\begin{tabular}{|c|c|c|}
\hline $\begin{array}{c}\text { Concurrence } \\
\text { Différenciation }\end{array}$ & $\begin{array}{c}\text { Concurrence } \\
\text { forte }\end{array}$ & $\begin{array}{c}\text { Concurrence } \\
\text { Oligopolistique }\end{array}$ \\
\hline $\begin{array}{c}\text { Différenciation verticale } \\
\text { entre labels }\end{array}$ & $\begin{array}{c}\text { Concurrence destructrice } \\
\text { entre labels }\end{array}$ & $\begin{array}{c}\text { Dominance d'un label ou } \\
\text { d'un petit groupe de labels }\end{array}$ \\
\hline $\begin{array}{c}\text { Différenciation horizontale } \\
\text { entre labels }\end{array}$ & $\begin{array}{c}\text { Multiplicité de labels } \\
\text { instables }\end{array}$ & $\begin{array}{c}\text { Coexistence de quelques } \\
\text { labels spécialisés }\end{array}$ \\
\hline
\end{tabular}

Source : (Hobeika et al., 2013) 
Sur la base de l'ensemble des éléments du tableau, nous avons quatre situations qui illustrent la dynamique concurrentielle qui se crée entre différents porteurs de labels et qui accompagne la structuration du marché selon le type de différenciation:

- Différenciation verticale et concurrence forte: il s'agit d'une situation d'instabilité potentiellement destructrice surtout en l'absence d'un acteur dominant ou d'une intervention extérieure de l'Etat.

- Différenciation verticale et concurrence oligopolistique: situation de dominance d'un label ou d'un groupe de labels avec des niveaux de qualité différents, suffisamment restreints pour assurer la viabilité de chacun.

- Différenciation horizontale et concurrence forte: une multiplicité de labels instables difficilement viables car de petite taille. La multiplicité des caractéristiques labellisées peut créer de la confusion chez les consommateurs.

- Différenciation horizontale et concurrence oligopolistique: les labels sont plus viables car moins nombreux et ont des parts de marché plus larges. Ils coexistent sur les segments respectifs des caractéristiques qu'ils ont choisies.

Nous avons pu, à travers ce tableau, démontrer le lien existant entre la différenciation et la labellisation. En effet, la forme de la différenciation est capable, dans certains cas, de détruire la concurrence entre les labels comme elle est capable de les maintenir en situation de coexistence. Ce lien affecte, bien évidemment, le comportement des consommateurs et son processus de prise de décision.

Aprés avoir définit les différents concepts clés de notre recherche, la présentation du processus méthodologique utilisé pour arriver aux résultats constitue la phase suivante.

\section{La méthodologie de recherche}

Cette partie a pour objectif de décrire le processus que nous avons adopté afin d'obtenir et d'apporter des réponses à notre problématique. Ainsi, notre méthodologie s'est basée sur deux axes principaux à savoir une revue de la littérature qui avait pour objectif de dégager, définir et maitriser les concepts clés de notre recherche à savoir les produits de terroir, les indications géographiques et la différenciation ainsi que le lien existant entre eux, et une étude qualitative à vocation exploratoire, qui avait pour objectif d'identifier les différents problèmes ou propriétés de l'indication géographique protégée notamment IGP Argane dans la différenciation de l'huile d'argan. vise à:

Pour Trudel, Simard et Vonarx (2007), toute recherche exploratoire

- $\quad$ Clarifier un problème préalablement défini, 
- $\quad$ Baliser une réalité et choisir les méthodes de collecte des données,

- Déterminer le sujet de recherche adéquat avant d'entamer une étude de plus grande ampleur,

- $\quad$ Extraire un minimum de connaissances pour effectuer la recherche,

- $\quad$ Sélectionner les sources de données pertinentes et efficaces.

Dans notre cas, il s’agit de faire ressortir les différentes variables qui justifient ou non le fonctionnement des indications géographiques comme outil de différenciation de l'huile d'argan notamment l'IGP Argane à travers des entretiens semi directifs auprès d'un échantillon de personnes qui représentent les différents acteurs ou les différentes parties prenantes représentant la ville d'Agadir et régions notamment les administrations publiques, les associations, les consommateurs (particuliers et professionnels) et les producteurs qu'ils soient des coopératives ou des sociétés privées, ces entretiens ont porté sur le cas de l'huile alimentaire et cosmétique bénéficiant ou non de l'IGP Argane et se situant dans la ville d'Agadir et régions. Cela nous permettra de faire d'analyse et de comprendre de manière pertinence et efficace le comportement de chacune des parties prenantes (leurs freins et motivations). Ces entretiens nous permettront de dresser un état de leur estimation de l'efficacité de l'IGP Argane comme outil de différenciation de l'huile d'argan. Nous serons alors en mesure d'identifier les points positifs et/ou négatifs des IG à travers l'exemple de l'IGP Argane, et d'analyser le processus de labellisation en examinant les obstacles et les succès résultant de cette démarche.

Pour Thietart (2007), le processus de la collecte des données s’arrête lorsque les dernières unités d'observations analysées n’apportent plus d'éléments nouveaux. Ce principe repose sur le fait que chaque unité d'information supplémentaire apporte un peu moins d'informations nouvelles que la précédente jusqu’à ne plus rien apporter. C'est ce qu'on appelle le concept de la saturation de l'information (ou la saturation théorique) définie par Glasser et Strauss (1997) cité par (Andreani et Conchon). Ainsi, nos entretiens ont porté sur 2 GIE (Groupement d'Intérêt Economique Targanine et UCFA) comprenant 29 coopératives, 10 consommateurs particuliers et 2 SPA appartenant à deux hôtels très connus à la ville d'Agadir , 2 revendeurs, 5 administrations publiques et 5 sociétés privées.

Dans le but de mener à bien notre étude, nos guides d'entretien (Voir Annexes) ont été administrés aux personnes susceptibles de détenir l'information notamment aux responsables des organisations en question (président de l'association ou de la coopérative, responsable qualité, responsable IGP, consommateur, responsable approvisionnement SPA, ...) avec qui nous avions pris des rendez-vous à l'avance par téléphone. La durée 
moyenne des entretiens a été de 30 à 40 minutes, les entretiens se sont déroulés au sein de :

- $\quad$ Points de vente des producteurs

- Hôtel SPA

- Salons professionnels

- Administrations

- $\quad$ Coopératives

\section{Résultats et discussion}

\section{Présentation et analyse des résultats}

Pour garantir un maximum de fiabilité et de validité dans notre étude, nous avons élaboré quatre guides d'entretien pour chacune des parties (voir annexes), dont un pour les producteurs détenant une IGP Argane, les administrations et les organisations concernées (ANCA, AMIGHA, PAMPAT, chambre de commerce, ODCO), un deuxième pour les producteurs non certifiés IGP, un troisième pour les consommateurs particuliers et revendeurs et un dernier pour les SPA.

Notre étude a pour but d'expliquer et d’interpréter les raisons du succès ou de l'échec de la démarche de labellisation des produits de terroir plus précisément l'huile d'argan par une IGP Argane. Pour cela, nous avons eu recours à la méthode d'analyse des données qualitatives appelée aussi l'analyse de contenu (Andreani et Conchon, 2005).

Tableau 3: Analyse des données qualitatives

\begin{tabular}{|c|c|}
\hline Analyse des données qualitatives & Interpretation des résultats \\
\hline Données du terrain & Problématique d'étude \\
\hline Analyse du contenu & Method Interprétative \\
\hline Description du materiel recueilli & $\begin{array}{c}\text { Elaboration des conclusions et des } \\
\text { enseignements à tirer } \\
\text { Etude de la signification des données }\end{array}$ \\
& Evaluation des pistes et solutions \\
\hline
\end{tabular}

Source: Andreani et Conchon, 2005

Cette méthode a été définie par son fondateur BERELSON (1952) comme « une technique de recherche pour la description objective, systématique et quantitative du contenu manifeste de la communication ». Pour ce faire, il convient de respecter un processus de trois étapes:

- $\quad$ Retranscrire les données qualitatives: manuellement, il s'agit de faire l'inventaire des informations recueillies et de les transformer en écrit (Auerbach et Silverstein, 2003) ;

- $\quad$ Coder les informations recueillies: manuellement aussi, la codification des informations, constitue un processus qui explore ligne par ligne, étape par étape, les textes des interviews ou des observations (Berg, 2001). Le codage des informations est soit ouvert soit fermé, le tabaleau suivant montre les caractéristiques de chacun des deux types de codage. 
Tableau 4: Grille de codage

\begin{tabular}{|c|c|}
\hline Codage ouvert & Codage fermé \\
\hline $\begin{array}{c}\text { Lecture ligne par ligne des données pour les } \\
\text { généraliser (processus d'abstraction) }\end{array}$ & $\begin{array}{c}\text { Lecture ligne par ligne des données et } \\
\text { codage en function des hypotheses de } \\
\text { recherché (processus de traduction) }\end{array}$ \\
\hline $\begin{array}{c}\text { Recherché d'ensemble similaires, classement et } \\
\text { comparaison }\end{array}$ & $\begin{array}{c}\text { Variables explicatives et variables à } \\
\text { expliquer établies de façon à priori }\end{array}$ \\
\hline $\begin{array}{c}\text { Codage des principales dimensions et codage } \\
\text { sélectif des idées centrales et répétitives }\end{array}$ & Codage des indicateurs de recherche \\
\hline
\end{tabular}

Source: Berg, (2001)

- $\quad$ Traiter les données: le traitement des données se fait de deux manières, soit sémantique ou statistique. La première consiste en le traitement manuel des données à travers une analyse empirique des idées, des mots, et de leurs significations alors que la seconde, utilise le traitement informatique en vue d'une analyse statistique des mots et des phrases (Andreani et Conchon, 2005).

Après avoir retranscrit, codé et traité les informations recueillies, elles se sont arrivées au stade de l'interprétation. Il s'agit d' " un diagnostic pas à pas des solutions partant de la description fidèle des interviews ou des observations en déterminant les conséquences du point de vue des choix stratégiques ou des concepts théoriques ». Il existe deux types d'interprétation (Andreani et Conchon, 2005):

- $\quad$ Une interprétation factuelle: Elle détermine par écrit le sens des données et commente les résultats en fonction des questions posées par l'enquête. Elle permet de faire un diagnostic des informations analysées et de les classer (Buber, Gadner, Richards, 2004) cité par (Andreani et Conchon, 2005.);

- Une interprétation subjective: elle nécessite l'intervention du chercheur ou du chargé de l'étude pour arbitrer une étude qui fournit un matériel incomplet pour en traduire les conclusions.

Figure 1: notre processus d'analyse de contenu et d'interprétation

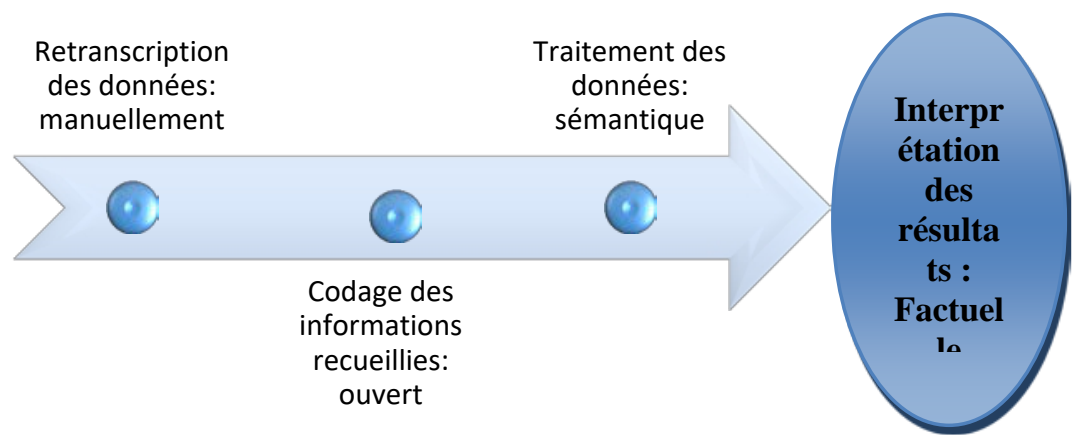

Source: travail personnel 
Le schéma ci-dessus montre notre processus d'analyse de contenu et d'interprétation des résultats, nous avons eu recours à la retranscription manuelle des données, puis au codage ouvert des informations recueillies pour arriver à traiter les données sémantiquement et finir par une interprétation factuelle des résultats.

\section{Interprétation des résultats:}

1) Pour les producteurs avec IGP:

Les producteurs qui détiennent le label IGP Argane avancent plusieurs motivations leur incitant à opter pour une telle stratégie de labellisation, ils justifient leur choix par le fait qu'ils veulent rester toujours compétitifs, et faire comme les autres, face à une situation de forte concurrence, ainsi, pour eux (les producteurs) le label est toujours un plus pour convaincre le client d'acheter le produit. Pour eux, la certification apparait comme une nécessité. En effet, pour survivre sur le marché concurrentiel et gagner de l'argent, les producteurs doivent s'inscrire dans une démarche de certification par une IGP et réaliser des profits, des bénéfices et des parts de marché.

Dans ce sens, l'exigence des clients, surtout étranger, constitue aussi une motivation derrière le choix de la stratégie de labellisation, car, le client est au centre de leurs préoccupations et il doit de ce fait être satisfait sur tous les niveaux.

L’engagement dans une stratégie de labellisation n’émane pas toujours de la pure volonté des producteurs, ils se trouvent obligés à l'adopter, car elle constitue une condition essentielle pour pouvoir assister aux salons nationaux et internationaux « elle est toujours demandée par l'ADA (Agence de Développement Agricole) pour compléter la procédure de participation aux salons et manifestations nationales et internationales »

Les producteurs ayant une IGP Argane affirment que la certification de la qualité par l’IGP Argane, s’est répercutée positivement sur la qualité et l'étiquetage de leurs produits pas plus (grâce notamment au respect du Cahier des Charges). Elle assure une certaine traçabilité et facilite l'audit, en plus elle donne une bonne image car elle contribue au développement social des populations de la région. Ainsi la raison avancée pour le choix de l’IGP Argane concerne leur notoriété.

Nous avons présenté les motivations qui ont poussé les producteurs à opter pour une stratégie de labellisation via une IGP Argane, cependant, ces mêmes producteurs expriment et attendent beaucoup de chose de ce label. Tantôt, des attentes propres à eux, tantôt des attentes pour le bien du produit de terroir marocain qui est l'huile d'argan.

Leurs attentes personnelles, consistent d'abord en l'interdiction de la vente en vrac, pour eux, cette pratique nuit à leurs activités et au produit lui- 
même, car la vente en vrac, entraine une baisse verticale des prix ce qui dévalorise également le produit. En suite, l’accréditation de l’organisme certificateur (qui s’appelle Normacert) constitue une attente majeure des producteurs avec IGP Argane, cette accréditation, permettra la reconnaissance de l'IGP Argane en Europe, ainsi, la vente en vrac, et l'exportation du produit non labellisé sera éventuellement interdite. Et enfin, l'unification des prix et la structuration du secteur, constitue le souhait principal des producteurs certifiés IGP Argane, ainsi, un comité de contrôle des prix et de la certification est recommandé pour assurer une certaine égalité entre les producteurs « il n’existe pas d'entente entre les producteurs qui ont une IGP Argane et ceux qui n’en ont pas, ni entre les sociétés privées et les coopératives, le secteur de la production et la commercialisation est en désordre total »

Quant aux attente liées au bien du produit lui-même, les producteurs certifiés souhaitent, d'une part, que l'IGP Argane contribue à la valorisation de ce produit de terroir qui n’existe qu’au Maroc « il faut qu'il soit un produit de LUXE », et d'autres part à sa protection comme patrimoine culturel national «c'est un produit 100\% marocain, personne n’a le droit de détruire son image »

2) Pour les producteurs qui n’ont pas un label IGP Argane, les freins liés à la labellisation de leurs produits sont:

Nous avons interrogé des producteurs de l'huile d’argan qui n’ont pas labellisé leurs produits sous une IGP Argane, pour comprendre les freins leur empêchant de s’inscrire dans une telle stratégie.

Contrairement aux producteurs certifiés, leurs concurrents non certifiés trouvent que le client n’est pas exigeant, car ils réalisent toujours de bons chiffres d'affaires et des ventes importantes. Pour eux, la labellisation est un coût supplémentaire du fait que le client ne la demande pas. Ils s'abstiennent à certifier leurs produits sous prétexte du pouvoir d'achat du client marocain, qui pour eux n’est pas prêt à payer un prix élevé rien que pour une certification de la qualité liée à l'origine.

Aussi, les petits producteurs de cette catégorie, notamment les coopératives, avancent que la labellisation de l'huile d'argan par une IGP Argane ne concerne que les producteurs qui font de l'exportation et qui ont un contact permanent avec les clients étrangers, souvent exigeants. Ils justifient leur choix également par les ressources financières limitées, dans ce sens, les coopératives, font face à une insuffisance financières "la certification IGP Argane est un luxe réservé uniquement aux grands producteurs qui détiennent les moyens financiers.,,

Les producteurs sans IGP Argane, avancent que la procédure de la labellisation est contraignante: elle nécessite le temps, l'effort, la patience et l'argent aussi, ils jugent que les procédures de la labellisation leur sont très 
contraignantes. Pour eux, l'huile d'argan n’a pas besoin de label pour se différencier, car c'est un produit qui n’existe qu'au Maroc et tous les consommateurs l'achètent de part le monde.

3) Pour les administrations

Les responsables des différentes administrations publiques que nous avons interviewées sont en unanimité sur le fait que l'IGP Argane est un outil pertinent pour la différenciation, la valorisation et la protection de l'huile d'argan, mais dans un contexte « favorable ».

Selon ces mêmes responsables, l'IGP Argane se heurte à plusieurs contraintes, se qui l'empêche de donner ses fruits et ses effets positifs. Pour eux, le manque de rigueur dans l'application et dans le contrôle de la certification, donne lieu à un contexte défavorable pour l'IGP Argane.

Ce contexte se caractérise par l'existence de la vente en vrac (que les producteurs avec IGP Argane souhaitent interdire) et l'existence du produit non certifié, constituent -pour la majorité de ces responsables-, le principal facteur de la dévalorisation du produit national puisque le produit non certifié circule partout et à des prix concurrentiels. Cette question des prix, rejoint l'idée du manque de l'entente entre les producteurs, qui mêmes au sein des salons professionnels internationaux et dans des pays étrangers ne sont pas d'accord sur les prix « on se met d'accord sur le prix de chacun des produits, mais quand ils sont dans leurs stands, ils veulent juste vendre »

Les différents représentants des administrations publiques, avancent que même avec une IGP Argane, les usurpations sont toujours possibles, notamment avec l'existence de la FAUSSE IGP Argane, il s'agit de fausses étiquettes à coller sur le produit falsifié destiné à l'export ce qui remet la traçabilité de la certification en question et nuit à la notoriété du produit de terroir marocain qui est dans notre cas l'huile d'argan.

Les administrations publiques, assument elles aussi la responsabilité devant «l'échec » de l'IGP Argane et avance que chacune des parties prenantes prend part de cette responsabilité. En effet, l'Etat marocain et le ministère de l'agriculture et de la pêche maritime (MAPM) avec ses directions et ses organismes chargés de la labellisation des produits de terroir, constituent la colonne vertébrale de la certification. Ainsi, le manque terrible de promotion, de communication et de vulgarisation de la labellisation et le Refus de la reconnaissance au niveau de l'Union Européenne (organisme certificateur non accrédité NORMACERT) constituent leurs principales erreurs «personne ne demande l'IGP. Ils ne savent pas c'est quoi une IGP ou un label »

Le consommateur marocain, est pour eux, également concerné par cet échec. Selon eux, de par sa nature, le consommateur marocain n'est pas prêt à payer plus pour certifier l'origine, pour lui « l'huile d'argan ne vient que de la ville d'Agadir et régions et de nulle part ailleurs». 
4) Pour les consommateurs de l'huile d'argan:

Pour l'huile d'argan cosmétique, nous avons interrogé les maisons de SPA de deux hôtels très connus à Agadir. Les responsables d'approvisionnement et de SPA insistent sur l'achat de l'huile certifiée, mais surtout BIO, "c'est une exigence, pour éviter les allergies de la peau car l'huile d'argan bio peut s'appliquer à tous les types de peaux sans aucun problème »; ils sont de ce fait, totalement satisfaits de leurs fournisseurs qui n’ont pas une IGP, et sont restés fidèles à eux « je suis satisfaite, pourquoi je vais changer mon fournisseur actuel »

Ils n’ont jamais utilisé ou acheté une huile labellisée IGP Argane, ils n'en connaissent pas (ce qui rejoint l'idée du manque de communication et de vulgarisation), ainsi, nous leur avons demandé (après avoir expliqué c'est quoi une IGP Argane et ses objectifs), si ils ont l'intention d'acheter une huile certifiée IGP Argane dans le futur, ils résument leurs critères d'achat en la qualité, le fait d'aider les femmes de la région et valoriser le produit marocain en général.

Pour la deuxième catégorie des consommateurs à savoir les particuliers, la majorité d'entre eux (citoyens: sauf quelques scientifiques et professionnels) ne sont pas au courant des indications géographiques protégées ni des labels en général exception faite au label bio qui bénéficie de la plus grande notoriété par rapport à l'AOP, IGP ou le Fair Trade. Pour la troisiéme catégorie des consommateurs, celle des revendeurs, ils n’achètent pas de l'huile certifiée IGP Argane, personne ne la demande ce qui rejoint l’idée précédante.

\section{Discussion des résultats}

On peut dire que la majorité des parties prenantes ont été déçues du label IGP Argane. En fait ce n'est pas le label en lui-même, mais c'est son contexte qui présente un handicap et une contrainte de grande envergure à son application et à l'atteinte de ses objectifs dits nobles objectifs, puisque les indications géographiques cherchent avant tout à assurer le développement économique et social des populations locales qui détiennent un savoir faire unique et exclusif à ces régions.

L'exemple de l'IGP Argane, montre qu'il y a encore des efforts à faire pour garantir la protection et la valorisation de ce produit artisanal qui est l'huile d'argan notamment au niveau de la reconnaissance de l'IGP Argane dans l'Union Européenne car selon (ILBERT et al., 2009) la plus importante des décisions relatives aux IG est leur reconnaissance, ainsi les autorités et les responsables de la certification doivent agir sur plusieurs aspects et qui sont:

- $\quad$ Promotion et communication de l'IGP Argane

- $\quad$ Le contrôle de l'IGP Argane: fausse étiquette IGP Argane 
- $\quad$ Facilitation de la procédure de certification

- $\quad$ Structuration du secteur: interdire la vente en vrac et l'exportation du produit non certifié

- Vulgarisation du label: faciliter la compréhension aux consommateurs

- $\quad$ La reconnaissance de l'IGP Argane au niveau de l'union européenne

Plusieurs produits ont fait face à des contraintes similaires que ceux qu'affronte l'huile d'argan IGP, l'exemple de l'oie de Baie du Febvre présente aussi des limites, en effet, ce produit s'est heurté à un ensemble de difficultés semblables à ceux de l'IGP Argane comme par exemple le manque de moyen financier pour s'imposer sur le marché ou l'absence de la coordination dans les actions collectives (Chazoule \& Lambert, 2007), ce qui démontre que l'IGP Argane ne constitue pas une exception.

Certes, il est évident que toute procédure de certification ou de labellisation fait face au début de son application à une multitude de contraintes et de freins, il convient alors de les dépasser par la suite à travers l'unification des efforts et la coordination entre les parties concernées pour atteindre les objectifs recherchés qui sont: la valorisation et la protection des produits de terroir.

\section{Conclusion}

Turgeon (2010) avance que l'abondance de produits standardisés venant de partout et de nulle part provoque un sentiment d'aliénation, d'incertitude et d'inquiétude chez le consommateur. Par contre, les produits de terroir, présentent une certaine assurance au niveau de la qualité et la santé et sont spécifique à leur région de production. Ils représentent une opportunité à ne pas rater pour les producteurs.

Actuellement, ces produits souffrent de pratiques déloyales et frauduleuses de la part des concurrents qui ne veulent que vendre, ainsi, ces produits doivent être valorisés, différenciés et protégés moyennant les SDOQ. Nous avons pris l'exemple de l'IGP Argane pour étudier la pertinence de ces SDOQ notamment les IG dans la différenciation des produits de terroir ; cas de l'IGP Argne.

Pour conclure, cet article, l'IGP Argane date de 2009. Son effet positif attendu n'est pas encore achevé. Plusieurs raisons le justifient et plusieurs acteurs en sont responsables. Notre étude a démontré que les IGP constituent un outil pertinent de différenciation des produits de terroir à condition que le contexte de ce label c'est-à-dire son environnement interne et externe soit favorable et assure une certaine fluidité des procédures et du contrôle.

Nous avons étudié la pertinence de cet outil à travers une étude qualitative auprès des parties prenantes concernées par la labellisation via 
une IGP Argane notamment les producteurs, les administrations et les consommateurs. La principale limite de notre travail réside au niveau des producteurs qui ne détiennent pas la certification IGP Argane et surtout les sociétés privées. En effet, les responsables de celles-ci dès qu’ils entendent parler de l'IGP Argane, refusent d'effectuer l'entretien et coupent la ligne directement, exception faite, aux grands groupes qui se sont imposés sur le marché national et international avec ou sans IGP Argane.

Au niveau des consommateurs, il est difficile de parler de perception du fait que la majorité entre eux ne sont pas informés de l'existence ou l'utilité de ces labels de l'origine et de la qualité, d'où un effort nécessaire à faire au niveau de la communication et la vulgarisation de l'intérêt de ces démarches de certification.

Les producteurs qui ont certifié leur huile d'argan, sont tous d'accord sur le fait que l'IGP Argane n'a pas influencé ni positivement ni négativement la promotion et le positionnement de leurs produits sur le marché national ou international, ils ne sont satisfaits que par le seul fait de pouvoir assister gratuitement à des salons nationaux et internationaux notamment les coopératives (dans lesquels le produit non certifié existe toujours) et l'amélioration de la qualité grâce au cahier des charges qui impose le respect des normes de l'hygiène et de la qualité.

Les administrations, quand à elles, font leurs efforts en espérant que cela aboutira aux objectifs escomptés dans le futur proche car l'huile d'argan est une mine d'or qu'il faut valoriser et protéger, c'est un produit déjà différencié dans le monde puisque il n’existe qu'au Maroc.

A travers cette étude exploratoire et ces réflexions nous avons dégagé plusieurs variables liées à la différenciation des produits de terroir et qui sont:
- Qualité
- Contrôle
- Communication
- Coordination
- Positionnement
Pour conclure, les Indications Géographiques constituent un outil pertinent pour la différenciation des produits de terroir, pour lesquelles il faut préparer déjà une base solide pour en récolter ses fruits.

\section{References:}

1. Aboudrare, A., Lybbert, T. J., \& Magnan, N. (2009). Le marché de l'huile d'Argan et son impact sur les ménages et la forêt. Bulletin Mensuels D'information et de Liaison Du Pntta, (037), 77-80. Retrieved from (http://www.agrimaroc.net/bul175.htm

2. ADS. (2015). Guide en Matière de Labellisation et de Certification. 
Retrieved from http://www.ads.gov.ma/

3. Allaire, G., Sylvander, B., Belletti, G., Marescotti, a, Barjolle, D., Thévenod-Mottet, E., \& Tregear, a. (2005). Les dispositifs fran $\{c ̧\}$ ais et europ $\{$ é\}ens de protection de la qualit\{é\} et de l’origine dans le contexte de l'OMC: justifications g\{é\}n\{é\}rales et contextes nationaux. Symposium International \{à\} Lyon Du, 9(i), 1-21.

4. Amajid, G., EL BAKKOURI, B., Souaf, M., \& Elwazani, Y. (2016). L'effet des produits de terroir sur l'attractivité et le développement de la région Souss Massa.

5. AMIGHA. (2009). Lettre d'information, 1-8.

6. Andreani, \& Conchon. (2005). METHODES D ' ANALYSE ET D ' INTERPRETATION DES ETUDES QUALITATIVES : ETAT DE L ' ART EN, (June 2016).

7. Arjaliès, D.-L., Hobeika, S., Ponsard, J.-P., \& Poret, S. (2013). Le rôle de la labellisation dans la construction d'un marché. Le cas de l'ISR en France. Revue Française de Gestion, 39, 93-107. http://doi.org/10.3166/rfg.236.93-107

8. Auerbach, C., \& Silverstein, L. B. (2003). Introducing qualitative hypothesis-generating research. Qualitative Data: An Introduction to Coding and Analysis, 1 - 9. Retrieved from https://www.questia.com/library/118079024/qualitative-data-anintroduction-to-coding-and-analysis

9. Barham, E. (2003). Translating terroir: The global challenge of French AOC labeling. Journal of Rural Studies, 19(1), 127-138. http://doi.org/10.1016/S0743-0167(02)00052-9

10. Bérard, L., \& Marchenay, P. (2007). Produits de Terroir Comprendre et agir.

11. Berg, B. L. (2001). Qualitative research methods for the social sciences. Qualitative Research (Vol. Seventh Ed). http://doi.org/10.2307/1317652

12. Boisvert, V., \& Caron, A. (2010). La conservation de la biodiversité: Un nouvel argument de différenciation des produits et de leur territoire d'origine. Geographie Economie Societe, 12(3), 307-328. http://doi.org/10.3166/ges.12.307-328

13. Cartier, S. (2004). Strates, 11. Retrieved from http://strates.revues.org/396

14. Chambolle, C., \& Eric, G.-H. (2003). Certification de la qualité par une AOC : un modèle d'analyse. Economie et Prévision, 159(3), 8391.

15. chambre de commerce et d'industrie Alsace. (2013). PRINCIPAUX LABELS , LOGOS et MARQUAGE EUROPEENS, 1-7.

16. Chazoule, C., \& Lambert, R. (2007). L'émergence des appellations 
d’origine au Québec. Naissance d'une nouvelle convention de qualité. Économie Rurale, 299(299), 24-41. http://doi.org/10.4000/economierurale.210

17. Courvoisier, F. H. F.-A. C. (2009). Quelle innovation pour les produits régionaux et les produits du terroir ?, 26-27.

18. Dekhili, S., \& Achabou, M. a. (2013). Pertinence d'une double labellisation biologique-écologique auprès des consommateurs. Une application au cas des œufs. Économie Rurale. Agricultures, ..., 336(Juillet-Aout), 41-59. Retrieved from http://economierurale.revues.org/4002

19. Dekhili, Lucie Sirieix, S. (2013). Importance des critères de choix de l'huile d'olive. La méthode Best Worst. Économie Rurale, 344. Retrieved from http://economierurale.revues.org/3727

20. Dumas, L., Menvielle, W., D.Perreault, J., \& Pettigrew, D. (2006). Terroirs, agrotourisme et marketing : le cas des fromages québécois. Téoros - Revue de Recherche En Tourisme, $n^{\circ}$ 25-1, 34-41.

21. Filippi, M., \& Triboulet, P. (2006). COORDINATION DES ACTEURS ET VALORISATION DE PRODUITS LIÉS À L'ORIGINE. LES SIGNES D'IDENTIFICATION COMME SIGNES D'EXCLUSION? Revue d'Économie Régionale \& Urbaine, 103 à 129. Retrieved from http://www.cairn.info/revue-d-economieregionale-et-urbaine-2006-1-page-103.htm

22. Fournier, S., Verdeaux, F., Avril, M., \& Durand, C. (2008). Le développement des indications géographiques au sud: attentes des acteurs locaux et fonctions jouées. Etudes de cas en Indonésie et en Ethiopie, (1), 1-13.

23. Hobeika, S., Ponssard, J.-P., \& Poret, S. (2013). Le rôle stratégique d'un label dans la formation d'un marché. Le cas de l'ISR en France.

24. ILBERT, H., TEKELIOGLU, Y., \& TOZANLI, S. (2009). Séminaire international: Les produits de terroir, les identifications géographiques et le développement local durable des pays méditerranéens. (F. M. Muruzabal, Ed.)Options méditerranéennes (2008th ed.). Antalya Turquie. Retrieved from www.ciheam.org/publications

25. Juillard, E. (1962). LA RÉGION : ESSAI DE DÉFINITION Author ( s ): Annales de Géographie, 387(387), 483-499.

26. Lagrange, L., Briand, H., \& Trognon, L. (2000). Importance économique des filières agro-alimentaires de produits sous signes officiels de qualité. Économie Rurale, 258, 6-18. http://doi.org/10.3406/ecoru.2000.5189

27. Lybbert, T. J., Barrett, C. B., \& Narjisse, H. (2002). Market-based conservation and local benefits: The case of argan oil in Morocco. 
Ecological

Economics,

41(1),

125-144.

http://doi.org/10.1016/S0921-8009(02)00020-4

28. MAPM. (2012). Situation de l'agriculture Marocaine.

29. Martine, F., Seyrevath, P., \& Brun, J.-M. (2009). Agriculture Biologique et Indications Géographiques Protégées : Le potentiel de si- gnes de qualité pour valoriser les produits locaux au Cambodge. In localiser les produits (pp. 1-8).

30. OMC. (1994). Accord sur les aspects des droits de propriété intellectuelle qui touchent au commerce (pp. 349-396). Retrieved from https://www.wto.org/french/docs_f/legal_f/27-trips.pdf

31. OMPI. (2013). Indications géographiques introduction (N952(F) ed.). Genève.

32. Prévost, P., Capitaine, M., Gautier-Pelissier, F., Michelin, Y., Jeanneaux, P., Fort, F., ... Josien, É. (2014). Le terroir, un concept pour l'action dans le développement des territoires. VertigO, (Volume 14 Numéro 1). http://doi.org/10.4000/vertigo.14807

33. Romagny, B. (2009). L'IGP Argane, entre patrimonialisation et marchandisation des ressources. Maghreb - Machrek (Vol. 202). http://doi.org/10.3917/machr.202.0085

34. Simenel, R., Michon, G., Auclair, L., Thomas, Y., Romagny, B., \& Guyon, M. (2009). L'argan : l'huile qui cache la forêt domestique De la valorisation du produit à la naturalisation de l'écosystème. Autrepart, 50(2), 51. http://doi.org/10.3917/autr.050.0051

35. Sylvander, B., \& Barjolle, D. (2003). Facteurs de succès des produits d’origine certifiée dans les filières agro-alimentaires en Europe: Marché, ressources et institutions.

36. Tekelioglu, Y., Tozanli, S., Ilbert, H., \& Çağatay, S. (2013). Indications Géographiques, dynamiques socio-économiques et patrimoine bio-culturel en Turquie et dans les pays méditerranéens.

37. Thietart, R.-A. (2007). Méthodes de recherche en management, 656.

38. Trudel, L., Simard, C., \& Vonarx, N. (2007). La recherche qualitative est-elle nécessairement exploratoire? Recherches Qualitatives, Hors Série(5), 38-45. Retrieved from http://www.researchgate.net/profile/Louis_Trudel/publication/24209 0642_La_recherche_qualitative_estelle_ncessairement_exploratoire/links/Odeec5303644e71997000000.p df

39. Turgeon, L. (2010). Les produits du terroir, version Québec. Ethnologie Française, 40(3), 477-486. http://doi.org/10.3917/ethn.103.0477 


\section{Annexes:}

\section{Guide d'entretien pour les producteurs ayant une IGP et les} administrations publiques:

Dans le cadre de nos études doctorales, nous réalisons une étude qualitative sur l'effet des Indications Géographiques comme outil de différenciation des produits de terroir cas de l'IGP Argane de la ville d'Agadir et régions.

1) Considérez-vous les IG comme outil pertinent de différenciation des produits de terroir ? Pourquoi ?

2) Qu'en est-il pour l'IGP Argane dans la ville d'Agadir et régions?

3) Quel est son impact sur la promotion de l'huile d'argan ?

4) Quelle est la contribution de L'IGP Argane dans le positionnement de l'huile d'argan?

5) Y a-t-il une différence entre avant et après l'IGP ?

6) Qu'attendez-vous de l'IGP ?

7) L'IGP est-elle arrivée à satisfaire vos attentes ? Pourquoi ?

\section{Guide d'entretien pour les producteurs sans certification IGP Argane}

Dans le cadre de nos études doctorales, nous réalisons une étude qualitative sur la pertinence des Indications Géographiques comme outil de différenciation des produits de terroir cas de l'IGP Argane de la ville d'Agadir et régions.

1- Quels sont les certifications (labels) que vous avez ?

2- $\quad$ Quelle est la certification la plus pertinence pour vous ?

3- Considérez-vous les IG comme outil pertinent de différenciation des produits de terroir?

4- Qu'en est-il pour l'IGP Argane?

5- Quels sont les freins qui vous empêchent de certifier votre produit par une IGP ?

6- $\quad$ Est-ce que votre produit est bien positionné actuellement sur le marché ?

7- $\quad$ Est vous satisfait de votre stratégie marketing actuelle ?

8- $\quad$ Pensez-vous à certifier votre produit par une IGP au futur ? pourquoi ?

\section{Guide d'entretien pour les consommateurs-particuliers}

Dans le cadre de nos études doctorales, nous réalisons une étude qualitative sur l'effet des Indications Géographiques comme outil de différenciation des produits de terroir cas de l'IGP Argane de la ville d'Agadir et régions.

1) Consommez-vous de l'huile d'argan ? Cosmétique ou alimentaire ?

2) Êtes-vous satisfait de l'huile que vous consommez actuellement? Pourquoi ?

3) Quels sont vos critères d'achat ?

4) Achetez-vous de l'huile d'argan certifiée ? Pourquoi ?

5) Connaissez-vous les certificats de qualité et d'origine ou labels ? 
6) Qu'attendez-vous des certificats de qualité et d'origine ?

7) Qu'en est-il pour l'IGP Argane ? Pourquoi ?

8) L'IGP Argane est-elle arrivée à satisfaire vos attentes par rapport au produit? Pourquoi ?

\section{Guide d'entretien pour les maisons de SPA}

Dans le cadre de nos études doctorales, nous réalisons une étude qualitative sur l'effet des Indications Géographiques comme outil de différenciation des produits de terroir cas de l'IGP Argane de la ville d'Agadir et régions.

1- $\quad$ Utilisez-vous l'huile d'argan dans votre SPA ? pourquoi ?

2- $\quad$ Quels sont vos critères d'achat pour l'huile d'argan ?

3- $\quad$ Achetez-vous des produits certifiés ? pourquoi

4- $\quad$ Qu'en est-il pour l'huile d'argan certifiée par une IGP Argane ?

5- $\quad$ Y a-t-il une différence entre l’huile certifiée par IGP et non certifiée ?

6- $\quad$ attendez-vous quelque chose de plus de l'IGP Argane ?

7- L'IGP Argane est-elle arrivée à satisfaire vos attentes par rapport au produit? Pourquoi ? 